\title{
Case Report and Review of the Literature
}

\section{Cervical tuberculous spondylitis associated with systemic lupus erythematosus}

\author{
M Berker*,1, B Atalay ${ }^{1}$, F Söylemezoğlu ${ }^{2}$, S Arıoğul ${ }^{3}$ and S Palaoğlu ${ }^{1}$ \\ ${ }^{1}$ Department of Neurosurgery, Hacettepe University School of Medicine, Sihhiye, Ankara, 06100, Turkey; \\ ${ }^{2}$ Department of Neuropathology, Hacettepe University School of Medicine, Sihhiye, Ankara, 06100, Turkey; \\ ${ }^{3}$ Department of Internal Medicine, Hacettepe University School of Medicine, Sihhiye, Ankara, 06100, Turkey
}

\begin{abstract}
Study design: A case report of cervical tuberculous spondylitis associated with systemic lupus erythematosus (SLE). Infection is a frequent problem in SLE, especially in patients hospitalised with the complications of the disease. Tuberculous spondylitis very rarely occurs in SLE patients, and cervical involvement has not been previously reported.

Case report: A 54-year-old female patient was admitted to our hospital with a complaint of neck pain radiating to her shoulder of 2 months' duration. The neurological examination was completely normal and radiological investigations revealed narrowing, angulation and destruction of the end plates of the 5th and 6th cervical vertebrae. She has received corticosteroid and colchicine treatment for the diagnosis of SLE during the last 10 years. The anterior cervical approach was used and pyogenic material was debrided from the C5-6 intervertebral space, and an otogenous bone graft with a Smith Robinson type fusion was performed.

Conclusion: High doses of corticosteroids are implicated as a risk factor for infection in SLE patients. Early diagnosis and appropriate medical and surgical treatment, as well as increased awareness of higher susceptibility to opportunistic infections, such as tuberculous spondylitis, are keystones for decreasing morbidity and mortality in patients with SLE.
\end{abstract}

Spinal Cord (2001) 39, 549-553

Keywords: tuberculous spondylitis; systemic lupus erythematosus (SLE); cervical vertebrae

\section{Introduction}

Tuberculosis of the spine is prevalent all over the world, and there is an increasing trend in developed and developing countries, parallel to the growing numbers of immunocompromised patients. There is a wide spectrum of nervous system problems in patients with systemic lupus erythematosus (SLE). Peripheral neuropathies have been observed in about $15 \%$ of patients with SLE. ${ }^{1}$ Infection is a frequent problem in SLE especially in patients hospitalised with the complications of the disease. The high frequency and unusual spectrum of infections can be attributed to the multiple disturbances of immune function in SLE. ${ }^{2}$ Central nervous system manifestations such as seizures are common, especially in younger patients. Paralysis may also develop following intracerebral haemorrhage or thrombosis. Transverse myelitis and sterile meningitis may be observed. Tuberculous spondylitis very rarely occurs in SLE patients. ${ }^{1,3,4}$

*Correspondence: M Berker, Birlik mah., 23. cadde, Simkent sitesi, BLok 3, Daire 13. Cankaya, 06610, Ankara, Turkey

\section{Case report}

A 54-year-old female patient was admitted to our hospital with a complaint of neck pain radiating to her shoulder of 2 months' duration. The neurological examination was completely normal and radiological investigations revealed narrowing, angulation and destruction of the end plates of the 5th and 6th cervical vertebrae (Figure 1). Magnetic resonance revealed hypointensity in the $\mathrm{C} 5$ and $\mathrm{C} 6$ corpus and contrast enhancement anterior to the C5-6 disc space (Figure 2).

She had been followed by the department of internal medicine with the diagnosis of SLE and administered corticosteroid and colchicine therapy. Her erythrocyte sedimentation rate was found to be $72 \mathrm{~mm} / \mathrm{h}$, tuberculin skin test was unreactive, acid-fast bacilli in sputum were negative, and bone scan was normal. The results of all screening tests related to malignancy and infection were negative, other than an elevated erythrocyte sedimentation rate.

The anterior cervical approach was used and yellow pus was drained at the level of the C5 longus colli 


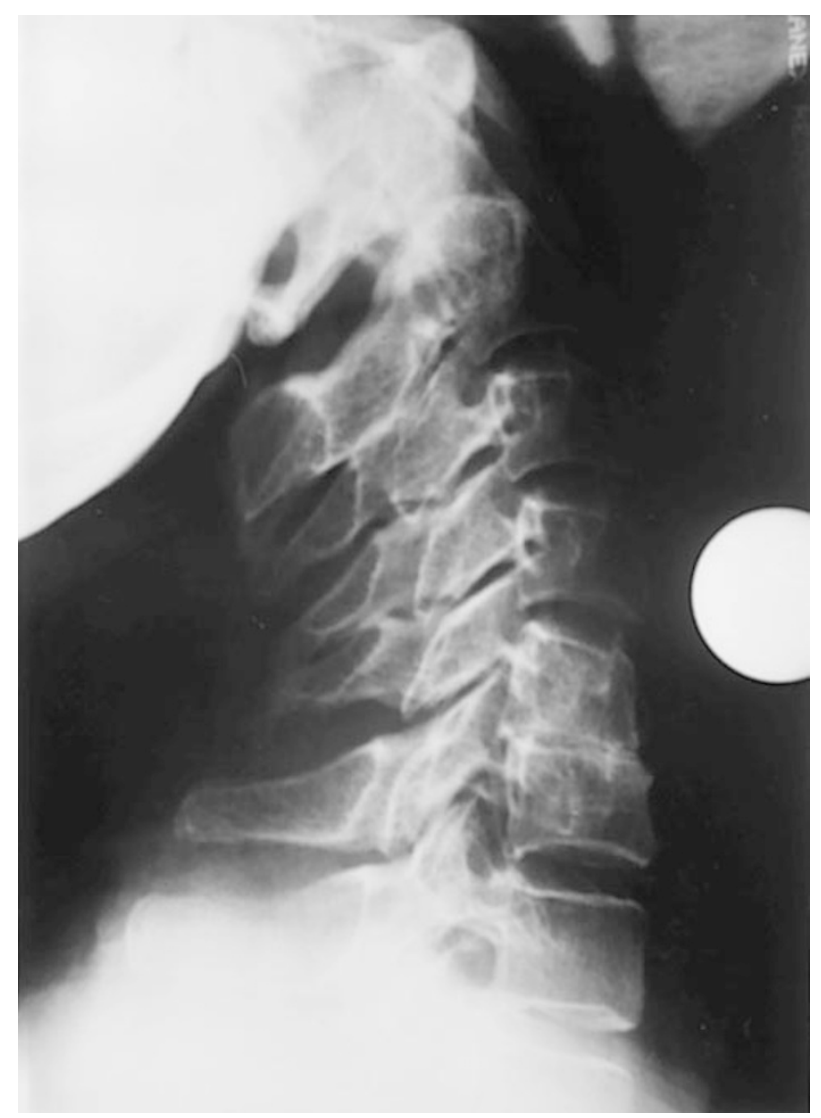

Figure 1 Preoperative cervical plain X-ray. Narrowing in C5-C6 intervertebral space

muscle. The pus and sequestrated disc materials were debrided from the C5-6 intervertebral space and fused by an otogenous bone graft with Smith Robinson type fusion (Figure 3).

Microbiological examination of the specimen with staining for acid-fast bacilli yielded positive results. PCR examination, after processing in the thermocycler (TTC 100 NJMJ Research Inc.), and the electrophoresis reaction was found to be positive. Mycobacterium tuberculosis was isolated with the Bactec 460 radiometric system. Histopathological examination revealed chronic granulomatous infection with caseous necrosis (Figure 4).

The patient was treated for tuberculous spondylitis, and antituberculosis chemotherapy, consisting of rifampicin, isoniazid, ethambutol and pyrazinamide, was started immediately. She was mobilised on the second postoperative day with a Philadelphia-type cervical collar; and discharged on the third postoperative day. After full-dose antituberculosis therapy for 2 months, ethambutol and pyrazinamide were discontinued. Rifampicin and isoniazid were continued for 9 months.

Two years later, her neurological examination was completely normal and radiological investigations did not reveal any abnormality.

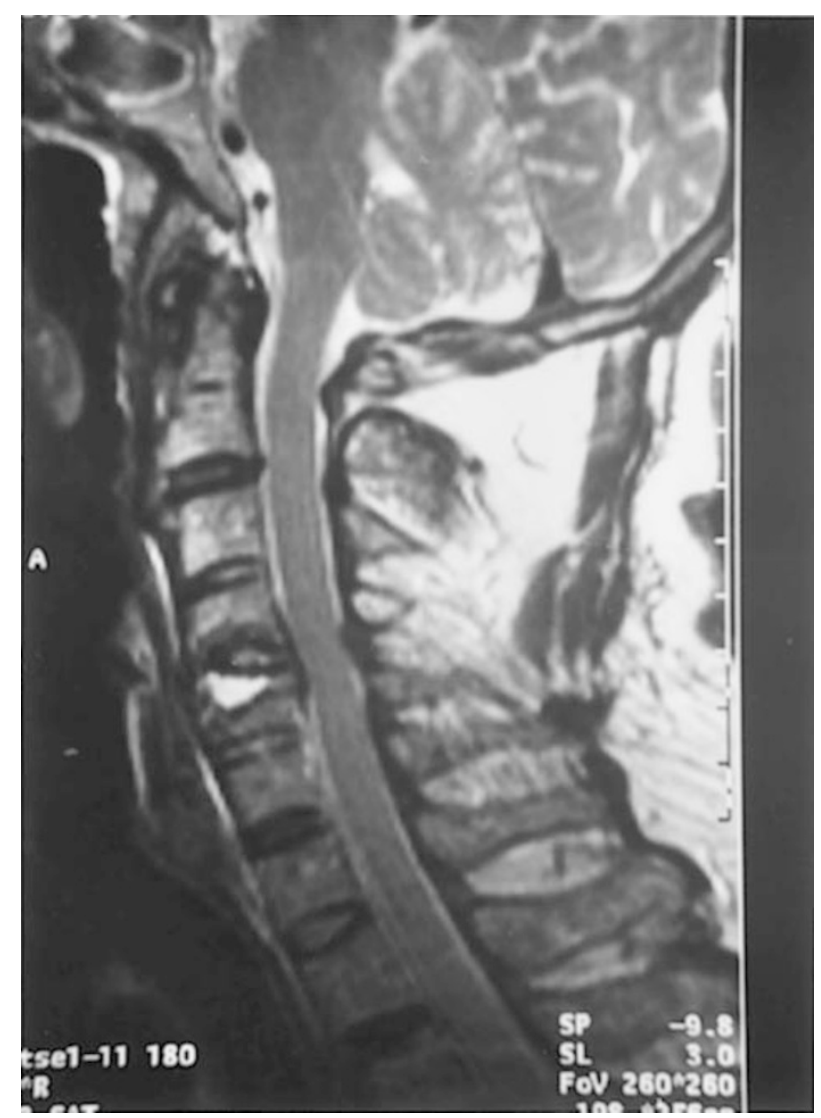

Figure 2 Preoperative T1 weighted MRI. Contrast enhancement anterior to the C5-C6 disc space

\section{Discussion}

Systemic lupus erythematosus is a disease of unknown aetiology characterised by inflammation in many different organ systems associated with the production of antibodies reactive with nuclear, cytoplasmic and cell membrane antigens. The immune hyperactivity that characterises SLE appears to derive from abnormal immune activation and loss of self-tolerance. An inherited defect in immune regulation may underlie the disorder in many individuals. Environmental agents that trigger the disease include foods, drugs, ultraviolet light and microorganisms. Inherited complement deficiency and other deficiencies may predispose patients to infections that induce disease activity. ${ }^{1,5}$

Individuals may have some, but not necessarily all, of the following signs and symptoms: fatigue, anaemia, fever, rashes, sun sensitivity, alopecia, arthritis, pericarditis, pleurisy, vasculitis and nephritis. ${ }^{1}$

The course is often unpredictable, with variable periods of exacerbations and remissions. About 85\% of the patients are women. Although the disease may occur at any age, most patients are between 10 and 50 years old, with the greatest clustering occurring between 20 and 40 . 


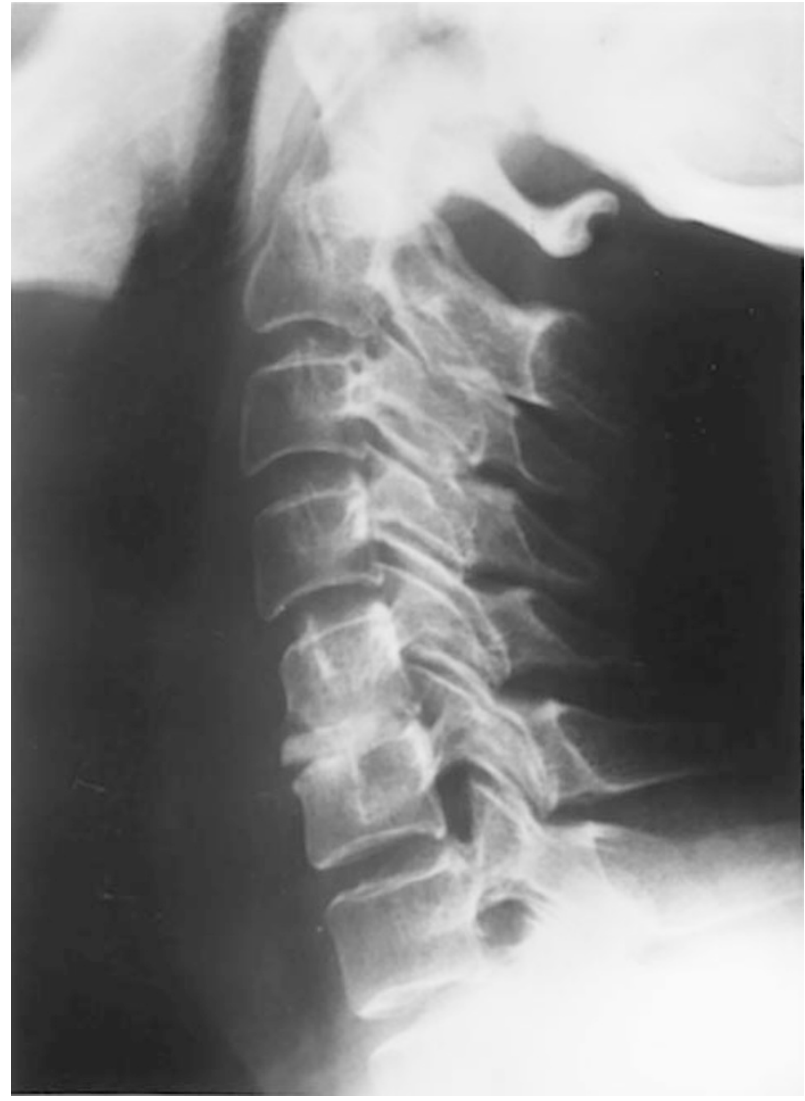

Figure 3 Postoperative cervical plain X-ray. Smith Robinson type fusion with an otogenous iliac graft

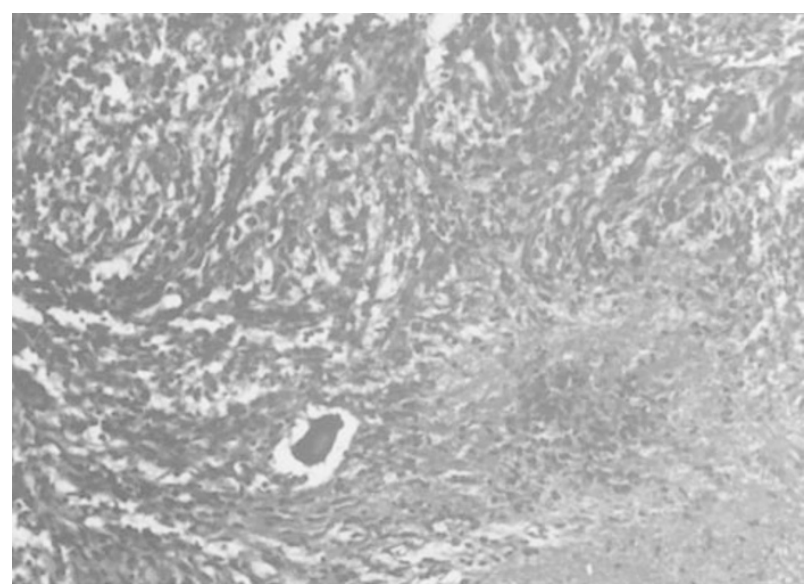

Figure 4 Note the presence of Langhans type giant cells at the border of caseous necrosis in granuloma $(\mathrm{HE} \times 200)$

The management of the SLE patient is often problematic and fraught with difficulties. High doses of corticosteroids are particularly implicated as a risk factor for infection, but the long-term toxicities of corticosteroids (cataracts, aseptic necrosis of bone, infections) must always be balanced against the benefits of continued vigorous therapy. ${ }^{2}$ The toxicity of immunosuppressive drugs is lower than that of prolonged high-dose corticosteroid therapy. ${ }^{1,5,6}$

Tuberculous spondylitis, also known as Pott's disease, is more common in developing or third-world countries. Tuberculous spondylitis most frequently involves the lower thoracic and upper lumbar vertebrae. Involvement of the cervical vertebrae is rare. $^{7-11}$ In our hospital, among the 20 patients who underwent surgical intervention for Pott's paraplegia, only $10 \%$ had cervical involvement. ${ }^{7}$ Usually more than one vertebra is affected, and in about $98 \%$ of cases the vertebral body is involved. Posterior vertebral elements are rarely involved. Psoas abscess is common. The destructive process usually starts in the disc space and involves the corpus. Sclerosis of the involved vertebral body may occur. ${ }^{11}$

Neurological deficits develop in $10-47 \%$ of patients, especially if the thoracic spine is involved. ${ }^{10}$ The infection itself rarely extends to the spinal canal; however, epidural granulation tissue, fibrosis or kyphotic bony deformity may cause cord compression. ${ }^{10}$

Tuberculin skin reaction is negative in about $14 \%$ of patients. ${ }^{12,13}$ It is unwise to use an arbitrary definition of a positive reaction in the diagnostic evaluation of a sick patient. Many factors may diminish the response in a nonspecific manner. These include viral infections or live virus vaccination; immunosuppression by disease, drugs, or steroids; malnutrition; overwhelming infection of any kind; and old age. ${ }^{14}$ To our knowledge, no epidemiological data have been reported with regard to the tuberculin test in tuberculous spondylitis patients with SLE. The test results can be expected to be negative in patients with inherited complement deficiency, abnormal immune activation and a history of therapeutic procedures for the SLE management including high-dosage corticosteroids and immunosuppressive drugs.

Rovensky et al pointed out the potential relationship of hyperprolactinaemia to the severity of SLE; however, we encountered no such finding. ${ }^{15}$

Plain X-ray changes take $2-8$ weeks from the onset of infection. The earliest changes are loss of the cortical endplate margins and the disc space height. ${ }^{16}$ In MRI, T1-weighted images show a confluent low signal from the vertebral bodies and intervertebral disc space. T2 weighted images show increased intensity from the involved vertebral body and disc space. ${ }^{17,18}$

Spinal brucellosis and pyogenic osteomyelitis caused by other bacteria (usually staphylococci), intervertebral disc herniation, actinomycosis, and metastatic lesions, as well as primary tumours of the reticuloendothelial system (such as multiple and solitary myelomas) must be considered in the differential diagnosis of tuberculous spondylitis. ${ }^{11}$ In Turkey, the most frequent aetiology of vertebral osteomyelitis is tuberculosis, and the second is brucellosis.

In the treatment of disease caused by $M$. tuberculosis susceptible to all agents, a 6-month course is 
effective and well tolerated. Isoniazid and rifampicin are used throughout the entire course of therapy, with pyrazinamide added during the first 2 months. Only patients with miliary, meningeal, or bone and joint disease require longer therapy (12 months). ${ }^{19}$ There is controversy regarding the duration of the antituberculous drug regimen for the management of tuberculous spondylitis, especially after the advent of specific antituberculous drugs and the radical surgery. Upadhyay et al have shown that a 6-month antituberculous chemotherapeutic regimen in conjunction with radical surgery is adequate for the management of tuberculosis of the spine, as it produced clinical and radiological results comparable with those of 9-month and 18 -month chemotherapeutic regimens. ${ }^{20}$

Friedman et al treated 64 patients with Pott's disease by prolonged administration of antituberculosis therapy, bedrest and braces. Spinal fusion was not performed, and laminectomy was reserved for eight patients with paresis. ${ }^{12}$ Lifeso et al, in their medical and surgical series of 107 patients with spinal tuberculosis, ${ }^{9}$ stated that their indications for an operation were neurological impairment, spinal instability, and failure of medical management.

Fang $e t a l^{21}$ reported the treatment of tuberculosis of the upper cervical spine ( $\mathrm{C} 1$ and $\mathrm{C} 2)$, and Hsu and Leong described the management of patients with lower cervical spine disease (C2 and C7). ${ }^{17}$ Shyam et al observed that tuberculosis was the commonest infectious disease among SLE patients in northern India. ${ }^{22}$ Victoria et al reported the rate of tuberculous osteomyelitis to be $7 \%$ in Filipino patients with systemic lupus erythematosus, pointing out the significant differences in mean SLE disease activity index and severity of disease index scores between those with limited tuberculosis versus those with extensive tuberculosis. ${ }^{23}$ Zvetina et al reported an SLE patient with Mycobacterium intracellulare infection of the shoulder and thoracolumbar spine and an associated psoas abscess, who was treated with steroids. ${ }^{5}$ Pirofsky et al reported an elderly patient who underwent steroid therapy for SLE and developed lower thoracic vertebral osteomyelitis caused by Mycobacterium tuberculosis. ${ }^{3}$ Pruitt et al presented the case of a SLE patient with steroid-dependent thoracic vertebral osteomyelitis secondary to Mycobacterium chelonae subspecies abscess. ${ }^{4}$

In the present case, we operated for correction of angulation and for histopathological diagnosis using an anterior cervical approach, and performed C5-C6 discectomy, debridement and graftings.

Our review suggests that this is the third reported case of SLE disease associated with TB spondylitis, and first involving the cervical spine. Tuberculosis must be kept in mind in patients with SLE, especially in developing countries.

Anti-tuberculous medication must be started as soon as possible, and the length of treatment should be at least 9 months. If there is neurological impairment, spinal instability and failure of medical management, then the choice of surgical treatment must be decompression and otogenous bone grafting. Early prognosis and appropriate treatment, as well as increased awareness of higher susceptibility to opportunistic infections, are keystones for decreasing morbidity and mortality in patients with SLE.

\section{References}

1 Steinberg AD. Systemic lupus erythematosus. In: Wyngaarden JB, Smith LH, Bennet JC (eds) Cecil Textbook of Medicine. 19th edn. W.B. Saunders: Philadelphia, 1992; pp. $1523-1530$.

2 Paton NI. Infections in systemic lupus erythematosus patients. Ann Acad Med Singapore 1997; 26: 694-700.

3 Pirofsky JG, Huang C-T, Waites KB. Spinal osteomyelitis due to Mycobacterium avium-intracellulare in an elderly man with steroid-induced osteoporosis. Spine 1993; 18: $1926-1932$.

4 Pruitt TC et al. Atypical myobacterial vertebral osteomyelitis in a steroid-dependent adolescent. Spine 1993; 18: $2553-2555$.

5 Zvetina JR, Demos TC, Rubinstein H. Mycobacterium intracellulare infection of the shoulder and spine in a patient with steroid-treated systemic lupus erythematosus. Skeletal Radiol 1982; 8: $111-113$.

6 Hellmann DB, Stone JH. Arthritis and musculoskeletal disorders. In: Tierney LM, McPhee SJ, Papadakis MA (eds). Current Medical Diagnosis and Treatment 40th edn. Lange MP: California, 2001, pp. 841-845.

7 Açıkgöz B et al. Surgery for progressive Pott's paraplegia (tuberculous paraplegia). Paraplegia 1991; 29: 537-541.

8 Kumar K. A clinical study and classification of posterior spinal tuberculosis. Int Orthop 1985; 9: 147-152.

9 Lifeso RM, Weaver P, Harder EH. Tuberculous spondylitis in adults. J Bone Joint Surg 1985; 67A: $1405-1413$.

10 Rocca HL. Spinal sepsis. In: Rothman RH, Simeone FA (eds). The Spine. 3rd edn. WB Saunders: Philadelphia, 1982; pp. $767-774$.

11 Tekkök IH et al. Brucellosis of the spine. Neurosurgery 1993; 33: $838-844$.

12 Friedman B. Chemotherapy of tuberculosis of the spine. J Bone Joint Surg 1966; 48A: 451-475.

13 Kaufman DM, Kaplan JG, Litman N. Infectious agents in spinal epidural abscesses. Neurology 1980; 30: 844-850.

14 Wolinsky E. Diseases due to mycobacteria. Tuberculosis. In: Wyngaarden JB, Smith LH, Bennet JC (eds). Cecil Textbook of Medicine. 19th edn. WB Saunders: Philadelphia, 1992, pp. 1733-1742.

15 Rovensky $\mathbf{J}$ et al. Relationship between endocrine, immune, and clinical variables in patients with systemic lupus erythematosus. J Rheumatol 1997; 24: 2330-2334.

16 Meschan I, Meschan RMF. Roentgen Signs in Diagnostic Imaging. V3. Spine and central nervous system. WB Saunders Company, 1985; pp. 79-81.

17 Hsu LCS, Leong JCY. Tuberculosis of the lower cervical spine (C2 to C7). J Bone Joint Surg 1984; 66B: $1-5$.

18 Modic MT et al. Vertebral osteomyelitis: assessment using MR. Radiology 1985; 157: 157-166.

19 Horsburgh R, Feldman S, Ridzon R. Practice guidelines for the treatment of tuberculosis. Clin Infect Dis 2000; 31: $633-639$ 
20 Upadhyay SS, Saji JM, Yau AC. Duration of antituberculosis chemotherapy in conjunction with radical surgery in the management of spinal tuberculosis. Spine 1996; 21: 1898 - 1903.

21 Fang D, Leong JCY, Fang HSY. Tuberculosis of the upper cervical spine. J Bone Joint Surg 1983; 65B: $47-50$
22 Shyam C, Malaviya AN. Infection related morbidity in systemic lupus erythematosus: a clinico-epidemiological study from northern India. Rheumatol Int 1996; 16: 1-3.

23 Victorio-Navarra ST, Dy EE, Arroyo CG, Torralba TP. Tuberculosis among Filipino patients with systemic lupus erythematosus. Semin Arthritis Rheum 1996; 26: 628634. 